\title{
Functional Space Consisted by Continuous Functions on Topological Space
}

\author{
Hiroshi Yamazaki \\ Nagano Prefectural Institute of Technology \\ Nagano, Japan \\ Yasunari Shidama \\ Karuizawa Hotch 244-1 \\ Nagano, Japan
}

Keiichi Miyajima

Ibaraki University

Ibaraki, Japan

\begin{abstract}
Summary. In this article, using the Mizar system [1, 2], first we give a definition of a functional space which is constructed from all continuous functions defined on a compact topological space [5]. We prove that this functional space is a Banach space [3. Next, we give a definition of a function space which is constructed from all continuous functions with bounded support. We also prove that this function space is a normed space.
\end{abstract}

MSC: 46E10 68V20

Keywords: continuous function space; compact topological space; Banach space MML identifier: COSP3, version: 8.1.11 5.65.1394

\section{Real Vector Space of Continuous Functions}

From now on $S$ denotes a non empty topological space, $T$ denotes a linear topological space, and $X$ denotes a non empty subset of the carrier of $S$.

Now we state the propositions:

(1) Let us consider a non empty topological space $X$, a non empty linear topological space $S$, functions $f, g$ from $X$ into $S$, and a point $x$ of $X$. Suppose $f$ is continuous at $x$ and $g$ is continuous at $x$. Then $f+g$ is continuous at $x$.

Proof: For every neighbourhood $G$ of $(f+g)(x)$, there exists a neighbourhood $H$ of $x$ such that $(f+g)^{\circ} H \subseteq G$. 
(2) Let us consider a non empty topological space $X$, a non empty linear topological space $S$, a function $f$ from $X$ into $S$, a point $x$ of $X$, and a real number $a$. If $f$ is continuous at $x$, then $a \cdot f$ is continuous at $x$.

Proof: For every neighbourhood $G$ of $(a \cdot f)(x)$, there exists a neighbourhood $H$ of $x$ such that $(a \cdot f)^{\circ} H \subseteq G$.

(3) Let us consider a non empty topological space $X$, a non empty linear topological space $S$, and functions $f, g$ from $X$ into $S$. If $f$ is continuous and $g$ is continuous, then $f+g$ is continuous.

Proof: For every point $x$ of $X, f+g$ is continuous at $x$.

(4) Let us consider a non empty topological space $X$, a non empty linear topological space $S$, a function $f$ from $X$ into $S$, and a real number $a$. If $f$ is continuous, then $a \cdot f$ is continuous. The theorem is a consequence of (2).

Let $S$ be a non empty topological space and $T$ be a non empty linear topological space. The continuous functions of $S$ and $T$ yielding a subset of RealVectSpace((the carrier of $S), T$ ) is defined by the term

(Def. 1) $\{f$, where $f$ is a function from the carrier of $S$ into the carrier of $T: f$ is continuous\}.

Let us observe that the continuous functions of $S$ and $T$ is non empty and functional.

Let us consider a non empty topological space $S$ and a non empty linear topological space $T$. Now we state the propositions:

(5) The continuous functions of $S$ and $T$ is linearly closed.

Proof: Set $W=$ the continuous functions of $S$ and $T$. For every vectors $v$, $u$ of RealVectSpace((the carrier of $S), T$ ) such that $v, u \in$ the continuous functions of $S$ and $T$ holds $v+u \in$ the continuous functions of $S$ and $T$. For every real number $a$ and for every vector $v$ of RealVectSpace((the carrier of $S), T$ ) such that $v \in W$ holds $a \cdot v \in W$.

(6) Lthe continuous functions of $S$ and $T$, Zero(the continuous functions of $S$ and $T$, RealVectSpace((the carrier of $S), T)$ ), Add(the continuous functions of $S$ and $T$, RealVectSpace((the carrier of $S), T)$ ), Mult(the continuous functions of $S$ and $T$, RealVectSpace((the carrier of $S), T))\rangle$ is a subspace of RealVectSpace((the carrier of $S), T)$.

Let $S$ be a non empty topological space and $T$ be a non empty linear topological space.

One can verify that $\langle$ the continuous functions of $S$ and $T$, Zero(the continuous functions of $S$ and $T$, RealVectSpace((the carrier of $S), T)$ ), Add(the continuous functions of $S$ and $T$, RealVectSpace((the carrier of $S), T)$ ), Mult (the continuous functions of $S$ and $T$, RealVectSpace((the carrier of $S), T))\rangle$ is Abelian, add- 
associative, right zeroed, right complementable, vector distributive, scalar distributive, scalar associative, and scalar unital.

The $\mathbb{R}$-vector space of continuous functions of $S$ and $T$ yielding a strict real linear space is defined by the term

(Def. 2) (the continuous functions of $S$ and $T$, Zero(the continuous functions of $S$ and $T$, RealVectSpace((the carrier of $S), T)$ ), Add(the continuous functions of $S$ and $T$, RealVectSpace((the carrier of $S), T))$, Mult(the continuous functions of $S$ and $T$, RealVectSpace((the carrier of $S), T))\rangle$.

Observe that the $\mathbb{R}$-vector space of continuous functions of $S$ and $T$ is constituted functions. Let $f$ be a vector of the $\mathbb{R}$-vector space of continuous functions of $S$ and $T$ and $v$ be an element of $S$. Let us note that the functor $f(v)$ yields a vector of $T$. Now we state the propositions:

(7) Let us consider a non empty topological space $S$, a non empty linear topological space $T$, and vectors $f, g, h$ of the $\mathbb{R}$-vector space of continuous functions of $S$ and $T$. Then $h=f+g$ if and only if for every element $x$ of $S, h(x)=f(x)+g(x)$. The theorem is a consequence of $(5)$.

(8) Let us consider a non empty topological space $S$, a non empty linear topological space $T$, vectors $f, h$ of the $\mathbb{R}$-vector space of continuous functions of $S$ and $T$, and a real number $a$. Then $h=a \cdot f$ if and only if for every element $x$ of $S, h(x)=a \cdot f(x)$. The theorem is a consequence of (5).

(9) Let us consider a non empty topological space $S$, and a non empty linear topological space $T$. Then $0_{\alpha}=($ the carrier of $S) \longmapsto 0_{T}$, where $\alpha$ is the $\mathbb{R}$-vector space of continuous functions of $S$ and $T$. The theorem is a consequence of (5).

Let $S$ be a non empty topological space and $T$ be a non empty linear topological space. Let us note that the carrier of the $\mathbb{R}$-vector space of continuous functions of $S$ and $T$ is functional.

\section{Real Vector Space of Continuous Functions (Norm Space VERSION)}

In the sequel $S, T$ denote real normed spaces and $X$ denotes a non empty subset of the carrier of $S$.

Now we state the proposition:

(10) Let us consider a point $x$ of $T$. Then (the carrier of $S$ ) $\longmapsto x$ is continuous on the carrier of $S$.

Let $S, T$ be real normed spaces. The continuous functions of $S$ and $T$ yielding a subset of RealVectSpace((the carrier of $S), T$ ) is defined by the term 
(Def. 3) $\{f$, where $f$ is a function from the carrier of $S$ into the carrier of $T: f$ is continuous on the carrier of $S\}$.

One can check that the continuous functions of $S$ and $T$ is non empty and functional.

Let us consider real normed spaces $S, T$. Now we state the propositions:

(11) The continuous functions of $S$ and $T$ is linearly closed.

Proof: Set $W=$ the continuous functions of $S$ and $T$. For every vectors $v$, $u$ of RealVectSpace((the carrier of $S), T$ ) such that $v, u \in$ the continuous functions of $S$ and $T$ holds $v+u \in$ the continuous functions of $S$ and $T$. For every real number $a$ and for every vector $v$ of RealVectSpace((the carrier of $S), T$ ) such that $v \in W$ holds $a \cdot v \in W$ by [4, (27)].

(12) Lthe continuous functions of $S$ and $T$, Zero(the continuous functions of $S$ and $T$, RealVectSpace((the carrier of $S), T)$ ), Add(the continuous functions of $S$ and $T$, RealVectSpace((the carrier of $S), T)$ ), Mult(the continuous functions of $S$ and $T$, RealVectSpace((the carrier of $S), T))\rangle$ is a subspace of RealVectSpace((the carrier of $S), T)$.

Let $S, T$ be real normed spaces. Observe that the continuous functions of $S$ and $T$, Zero(the continuous functions of $S$ and $T$, RealVectSpace((the carrier of $S), T)$ ), Add(the continuous functions of $S$ and $T$, RealVectSpace((the carrier of $S), T)$ ), Mult(the continuous functions of $S$ and $T$, RealVectSpace((the carrier of $S), T)$ ) $\rangle$ is Abelian, add-associative, right zeroed, right complementable, vector distributive, scalar distributive, scalar associative, and scalar unital.

The $\mathbb{R}$-vector space of continuous functions of $S$ and $T$ yielding a strict real linear space is defined by the term

(Def. 4) (the continuous functions of $S$ and $T$, Zero(the continuous functions of $S$ and $T$, RealVectSpace((the carrier of $S), T)$ ), Add(the continuous functions of $S$ and $T$, RealVectSpace((the carrier of $S), T)$ ), Mult(the continuous functions of $S$ and $T$, RealVectSpace $(($ the carrier of $S), T))\rangle$.

Note that the $\mathbb{R}$-vector space of continuous functions of $S$ and $T$ is constituted functions.

Let $f$ be a vector of the $\mathbb{R}$-vector space of continuous functions of $S$ and $T$ and $v$ be an element of $S$. One can check that the functor $f(v)$ yields a vector of $T$. Now we state the propositions:

(13) Let us consider real normed spaces $S, T$, and vectors $f, g, h$ of the $\mathbb{R}$ vector space of continuous functions of $S$ and $T$. Then $h=f+g$ if and only if for every element $x$ of $S, h(x)=f(x)+g(x)$. The theorem is a consequence of (11).

(14) Let us consider real normed spaces $S, T$, vectors $f, h$ of the $\mathbb{R}$-vector space of continuous functions of $S$ and $T$, and a real number $a$. Then 
$h=a \cdot f$ if and only if for every element $x$ of $S, h(x)=a \cdot f(x)$. The theorem is a consequence of (11).

Let us consider real normed spaces $S, T$. Now we state the propositions:

(15) The $\mathbb{R}$-vector space of continuous functions of $S$ and $T$ is a subspace of RealVectSpace((the carrier of $S), T)$.

(16) $0_{\alpha}=($ the carrier of $S) \longmapsto 0_{T}$, where $\alpha$ is the $\mathbb{R}$-vector space of continuous functions of $S$ and $T$. The theorem is a consequence of (11).

Let $S, T$ be real normed spaces and $f$ be an object. Assume $f \in$ the continuous functions of $S$ and $T$. The functor PartFuncs $(f, S, T)$ yielding a function from $S$ into $T$ is defined by

(Def. 5) $\quad i t=f$ and $i t$ is continuous on the carrier of $S$.

\section{Normed Topological Linear Space}

We consider normed real linear topological structures which extend real linear topological structures and normed structures and are systems

〈a carrier, a zero, an addition, an external multiplication,

$$
\text { a topology, a norm > }
$$

where the carrier is a set, the zero is an element of the carrier, the addition is a binary operation on the carrier, the external multiplication is a function from $\mathbb{R} \times$ (the carrier) into the carrier, the topology is a family of subsets of the carrier, the norm is a function from the carrier into $\mathbb{R}$.

Let $X$ be a non empty set, $O$ be an element of $X, F$ be a binary operation on $X, G$ be a function from $\mathbb{R} \times X$ into $X, T$ be a family of subsets of $X$, and $N$ be a function from $X$ into $\mathbb{R}$. Observe that $\langle X, O, F, G, T, N\rangle$ is non empty and there exists a normed real linear topological structure which is strict and non empty.

Let $X$ be a non empty normed real linear topological structure. We say that $X$ is normed structure if and only if

(Def. 6) there exists a real normed space $R$ such that $R=$ the normed structure of $X$ and the topology of $X=$ the topology of TopSpaceNorm $R$.

One can verify that there exists a non empty normed real linear topological structure which is strict, add-continuous, mult-continuous, topological spacelike, Abelian, add-associative, right zeroed, right complementable, vector distributive, scalar distributive, scalar associative, scalar unital, discernible, reflexive, real normed space-like, normed structure, and $T_{2}$. 
A normed linear topological space is a strict, add-continuous, mult-continuous, topological space-like, Abelian, add-associative, right zeroed, right complementable, vector distributive, scalar distributive, scalar associative, scalar unital, discernible, reflexive, real normed space-like, normed structure, $T_{2}$, non empty normed real linear topological structure. Now we state the propositions:

(17) Every normed linear topological space is a linear topological space.

(18) Every normed linear topological space is a real normed space.

(19) Let us consider a normed linear topological space $X$, and a real normed space $R$. Suppose $R=$ the normed structure of $X$. Let us consider points $x, y$ of $X$, points $x_{1}, y_{1}$ of $R$, and a real number $a$. Suppose $x_{1}=x$ and $y_{1}=y$. Then

(i) $x+y=x_{1}+y_{1}$, and

(ii) $a \cdot x=a \cdot x_{1}$, and

(iii) $x-y=x_{1}-y_{1}$, and

(iv) $\|x\|=\left\|x_{1}\right\|$.

Let us consider a normed linear topological space $X$, a sequence $S$ of $X$, and a point $x$ of $X$. Now we state the propositions:

(20) $S$ is convergent to $x$ if and only if for every real number $r$ such that $0<r$ there exists a natural number $m$ such that for every natural number $n$ such that $m \leqslant n$ holds $\|S(n)-x\|<r$. The theorem is a consequence of (19).

(21) $S$ is convergent and $x=\lim S$ if and only if for every real number $r$ such that $0<r$ there exists a natural number $m$ such that for every natural number $n$ such that $m \leqslant n$ holds $\|S(n)-x\|<r$. The theorem is a consequence of $(20)$.

(22) Let us consider a normed linear topological space $X$, and a sequence $S$ of $X$. Suppose $S$ is convergent. Let us consider a real number $r$. Suppose $0<r$. Then there exists a natural number $m$ such that for every natural number $n$ such that $m \leqslant n$ holds $\|S(n)-\lim S\|<r$. The theorem is a consequence of (20).

(23) Let us consider a normed linear topological space $X$, and a subset $V$ of $X$. Then $V$ is open if and only if for every point $x$ of $X$ such that $x \in V$ there exists a real number $r$ such that $r>0$ and $\{y$, where $y$ is a point of $X:\|x-y\|<r\} \subseteq V$. The theorem is a consequence of (19).

Let us consider a normed linear topological space $X$, a point $x$ of $X$, a real number $r$, and a subset $V$ of $X$. Now we state the propositions: 
(24) If $V=\{y$, where $y$ is a point of $X:\|x-y\|<r\}$, then $V$ is open. The theorem is a consequence of (19).

(25) Suppose $V=\{y$, where $y$ is a point of $X:\|x-y\| \leqslant r\}$. Then $V$ is closed. The theorem is a consequence of (19).

Now we state the propositions:

(26) Let us consider a normed linear topological space $X$, a real normed space $R$, a sequence $t$ of $X$, and a sequence $s$ of $R$. Suppose $R=$ the normed structure of $X$ and $t=s$ and $t$ is convergent. Then

(i) $s$ is convergent, and

(ii) $\lim s=\lim t$.

The theorem is a consequence of (22) and (19).

(27) Let us consider a normed linear topological space $X$, a real normed space $R$, a sequence $s$ of $X$, and a sequence $t$ of $R$. Suppose $R=$ the normed structure of $X$ and $s=t$. Then $s$ is convergent if and only if $t$ is convergent. The theorem is a consequence of (26), (19), and (21).

(28) Let us consider a normed linear topological space $X$, and a subset $V$ of $X$. Then $V$ is closed if and only if for every sequence $s_{1}$ of $X$ such that $\operatorname{rng} s_{1} \subseteq V$ and $s_{1}$ is convergent holds $\lim s_{1} \in V$. The theorem is a consequence of (26) and (27).

(29) Let us consider a normed linear topological space $X$, a real normed space $R$, a subset $V$ of $X$, and a subset $W$ of $R$. Suppose $R=$ the normed structure of $X$ and the topology of $X=$ the topology of TopSpaceNorm $R$ and $V=W$. Then $V$ is closed if and only if $W$ is closed. The theorem is a consequence of (27), (26), and (28).

(30) Let us consider a normed linear topological space $X$, a subset $V$ of $X$, and a point $x$ of $X$. Then $V$ is a neighbourhood of $x$ if and only if there exists a real number $r$ such that $r>0$ and $\{y$, where $y$ is a point of $X:\|y-x\|<r\} \subseteq V$. The theorem is a consequence of (23) and (24).

(31) Let us consider a normed linear topological space $X$, and a subset $V$ of $X$. Then $V$ is compact if and only if for every sequence $s_{1}$ of $X$ such that rng $s_{1} \subseteq V$ there exists a sequence $s_{2}$ of $X$ such that $s_{2}$ is subsequence of $s_{1}$ and convergent and $\lim s_{2} \in V$. The theorem is a consequence of (27) and (26).

(32) Let us consider a normed linear topological space $X$, a real normed space $R$, a subset $V$ of $X$, and a subset $W$ of $R$. Suppose $R=$ the normed structure of $X$ and the topology of $X=$ the topology of TopSpaceNorm $R$ and $V=W$. Then $V$ is compact if and only if $W$ is compact. The theorem is a consequence of (31), (26), and (27). 


\section{Real Norm Space of Continuous Functions}

Now we state the propositions:

(33) Let us consider sets $X, X_{1}$, a real normed space $S$, and a partial function $f$ from $S$ to $\mathbb{R}$. Suppose $f$ is continuous on $X$ and $X_{1} \subseteq X$. Then $f$ is continuous on $X_{1}$.

Proof: $f\left\lceil X_{1}\right.$ is continuous in $r$.

(34) Let us consider a non empty, compact topological space $S$, a normed linear topological space $T$, and a set $x$. Suppose $x \in$ the continuous functions of $S$ and $T$. Then $x \in \operatorname{BdFuncs}(($ the carrier of $S), T)$.

(35) Let us consider a non empty, compact topological space $S$, and a normed linear topological space $T$. Then the $\mathbb{R}$-vector space of continuous functions of $S$ and $T$ is a subspace of the set of bounded real sequences from the carrier of $S$ into $T$. The theorem is a consequence of (34) and (5).

Let $S$ be a non empty, compact topological space and $T$ be a normed linear topological space. The continuous functions norm of $S$ and $T$ yielding a function from the continuous functions of $S$ and $T$ into $\mathbb{R}$ is defined by the term

(Def. 7) BdFuncsNorm((the carrier of $S), T) \Gamma$ (the continuous functions of $S$ and $T)$.

The $\mathbb{R}$-norm space of continuous functions of $S$ and $T$ yielding a strict normed structure is defined by the term

(Def. 8) (the continuous functions of $S$ and $T$, Zero(the continuous functions of $S$ and $T$, RealVectSpace((the carrier of $S), T)$ ), Add(the continuous functions of $S$ and $T$, RealVectSpace((the carrier of $S), T)$ ), Mult(the continuous functions of $S$ and $T$, RealVectSpace((the carrier of $S), T)$ ), the continuous functions norm of $S$ and $T\rangle$.

One can check that the $\mathbb{R}$-norm space of continuous functions of $S$ and $T$ is non empty.

Now we state the propositions:

(36) Let us consider a non empty, compact topological space $S$, a normed linear topological space $T$, a point $x$ of the $\mathbb{R}$-norm space of continuous functions of $S$ and $T$, and a point $y$ of the real normed space of bounded functions from the carrier of $S$ into $T$. If $x=y$, then $\|x\|=\|y\|$.

(37) Let us consider a non empty, compact topological space $S$, a normed linear topological space $T$, a point $f$ of the $\mathbb{R}$-norm space of continuous functions of $S$ and $T$, and a function $g$ from $S$ into $T$. Suppose $f=g$. Let us consider a point $t$ of $S$. Then $\|g(t)\| \leqslant\|f\|$. The theorem is a consequence of (34). 
(38) Let us consider a non empty, compact topological space $S$, a normed linear topological space $T$, points $x_{1}, x_{2}$ of the $\mathbb{R}$-norm space of continuous functions of $S$ and $T$, and points $y_{1}, y_{2}$ of the real normed space of bounded functions from the carrier of $S$ into $T$. If $x_{1}=y_{1}$ and $x_{2}=y_{2}$, then $x_{1}+x_{2}=y_{1}+y_{2}$. The theorem is a consequence of (5).

(39) Let us consider a non empty, compact topological space $S$, a normed linear topological space $T$, a real number $a$, a point $x$ of the $\mathbb{R}$-norm space of continuous functions of $S$ and $T$, and a point $y$ of the real normed space of bounded functions from the carrier of $S$ into $T$. If $x=y$, then $a \cdot x=a \cdot y$. The theorem is a consequence of (5).

Let $S$ be a non empty, compact topological space and $T$ be a normed linear topological space. One can verify that the $\mathbb{R}$-norm space of continuous functions of $S$ and $T$ is non empty, right complementable, Abelian, add-associative, right zeroed, vector distributive, scalar distributive, scalar associative, and scalar unital.

Let us consider a non empty, compact topological space $S$ and a normed linear topological space $T$. Now we state the propositions:

(40) (The carrier of $S$ ) $0_{T}=0_{\alpha}$, where $\alpha$ is the $\mathbb{R}$-norm space of continuous functions of $S$ and $T$. The theorem is a consequence of (9).

(41) $0_{\alpha}=0_{\beta}$, where $\alpha$ is the $\mathbb{R}$-norm space of continuous functions of $S$ and $T$ and $\beta$ is the real normed space of bounded functions from the carrier of $S$ into $T$. The theorem is a consequence of (40).

Let us consider a non empty, compact topological space $S$, a normed linear topological space $T$, and a point $F$ of the $\mathbb{R}$-norm space of continuous functions of $S$ and $T$. Now we state the propositions:

(42) $0 \leqslant\|F\|$. The theorem is a consequence of (34).

(43) If $F=0_{\alpha}$, then $0=\|F\|$, where $\alpha$ is the $\mathbb{R}$-norm space of continuous functions of $S$ and $T$. The theorem is a consequence of (34) and (40).

(44) Let us consider a non empty, compact topological space $S$, a normed linear topological space $T$, points $F, G, H$ of the $\mathbb{R}$-norm space of continuous functions of $S$ and $T$, and functions $f, g, h$ from $S$ into $T$. Suppose $f=F$ and $g=G$ and $h=H$. Then $H=F+G$ if and only if for every element $x$ of $S, h(x)=f(x)+g(x)$. The theorem is a consequence of (7).

(45) Let us consider a real number $a$, a non empty, compact topological space $S$, a normed linear topological space $T$, points $F, G$ of the $\mathbb{R}$-norm space of continuous functions of $S$ and $T$, and functions $f, g$ from $S$ into $T$. Suppose $f=F$ and $g=G$. Then $G=a \cdot F$ if and only if for every element $x$ of $S, g(x)=a \cdot f(x)$. The theorem is a consequence of (8).

(46) Let us consider a real number $a$, a non empty, compact topological space 
$S$, a normed linear topological space $T$, and points $F, G$ of the $\mathbb{R}$-norm space of continuous functions of $S$ and $T$. Then

(i) $\|F\|=0$ iff $F=0_{\alpha}$, and

(ii) $\|a \cdot F\|=|a| \cdot\|F\|$, and

(iii) $\|F+G\| \leqslant\|F\|+\|G\|$,

where $\alpha$ is the $\mathbb{R}$-norm space of continuous functions of $S$ and $T$. The theorem is a consequence of (34), (38), (36), (41), and (39).

Let $S$ be a non empty, compact topological space and $T$ be a normed linear topological space. Let us observe that the $\mathbb{R}$-norm space of continuous functions of $S$ and $T$ is reflexive, discernible, and real normed space-like.

Now we state the propositions:

(47) Let us consider a non empty, compact topological space $S$, a normed linear topological space $T$, points $x_{1}, x_{2}$ of the $\mathbb{R}$-norm space of continuous functions of $S$ and $T$, and points $y_{1}, y_{2}$ of the real normed space of bounded functions from the carrier of $S$ into $T$. If $x_{1}=y_{1}$ and $x_{2}=y_{2}$, then $x_{1}-x_{2}=y_{1}-y_{2}$. The theorem is a consequence of (39) and (38).

(48) Let us consider a non empty, compact topological space $S$, a normed linear topological space $T$, points $F, G, H$ of the $\mathbb{R}$-norm space of continuous functions of $S$ and $T$, and functions $f, g, h$ from $S$ into $T$. Suppose $f=F$ and $g=G$ and $h=H$. Then $H=F-G$ if and only if for every element $x$ of $S, h(x)=f(x)-g(x)$. The theorem is a consequence of (44).

(49) Let us consider a non empty topological space $S$, a normed linear topological space $T$, a sequence $H$ of partial functions from the carrier of $S$ into the carrier of $T$, and a function $L_{1}$ from $S$ into $T$. Suppose $H$ is uniform-convergent on the carrier of $S$ and for every natural number $n$, there exists a function $H_{1}$ from $S$ into $T$ such that $H_{1}=H(n)$ and $H_{1}$ is continuous and $L_{1}=\lim _{\alpha} H$. Then $L_{1}$ is continuous, where $\alpha$ is the carrier of $S$.

Proof: For every point $x$ of $S, L_{1}$ is continuous at $x$ by (30), [7, (33),(11)].

(50) Let us consider a non empty, compact topological space $S$, a normed linear topological space $T$, and a subset $Y$ of the carrier of the real normed space of bounded functions from the carrier of $S$ into $T$. Suppose $Y=$ the continuous functions of $S$ and $T$. Then $Y$ is closed. The theorem is a consequence of (49).

(51) Let us consider a non empty, compact topological space $S$, and a normed linear topological space $T$. Suppose $T$ is complete. Let us consider a sequence $s_{3}$ of the $\mathbb{R}$-norm space of continuous functions of $S$ and $T$. 
If $s_{3}$ is Cauchy sequence by norm, then $s_{3}$ is convergent. The theorem is a consequence of (34), (47), (36), and (50).

(52) Let us consider a non empty, compact topological space $S$, and a normed linear topological space $T$. Suppose $T$ is complete. Then the $\mathbb{R}$-norm space of continuous functions of $S$ and $T$ is complete. The theorem is a consequence of (51).

\section{Some Properties of Support}

Let $X$ be a zero structure and $f$ be a (the carrier of $X$ )-valued function. The functor support $f$ yielding a set is defined by

(Def. 9) for every object $x, x \in$ it iff $x \in \operatorname{dom} f$ and $f_{/ x} \neq 0_{X}$.

Now we state the proposition:

(53) Let us consider a zero structure $X$, and a (the carrier of $X$ )-valued function $f$. Then support $f \subseteq \operatorname{dom} f$.

Let $X$ be a non empty topological space, $T$ be a real linear space, and $f$ be a function from $X$ into $T$. One can verify that the functor support $f$ yields a subset of $X$. Now we state the propositions:

(54) Let us consider a non empty topological space $X$, a real linear space $T$, and functions $f, g$ from $X$ into $T$. Then $\operatorname{support}(f+g) \subseteq \operatorname{support} f \cup$ support $g$.

(55) Let us consider a non empty topological space $X$, a real linear space $T$, a function $f$ from $X$ into $T$, and a real number $a$. Then $\operatorname{support}(a \cdot f) \subseteq$ support $f$.

\section{Space of Real-valued Continuous Functionals with Bounded SUPPORT}

Let $X$ be a non empty topological space and $T$ be a normed linear topological space. The functor $\mathrm{C}_{0}$ Functions $(X, T)$ yielding a non empty subset of RealVectSpace( (the carrier of $X), T$ ) is defined by the term

(Def. 10) $\{f$, where $f$ is a function from the carrier of $X$ into the carrier of $T: f$ is continuous and there exists a non empty subset $Y$ of $X$ such that $Y$ is compact and support $f \subseteq Y\}$.

Now we state the propositions:

(56) Let us consider a non empty topological space $X$, a normed linear topological space $T$, and elements $v, u$ of RealVectSpace((the carrier of $X), T)$. 
Suppose $v, u \in \mathrm{C}_{0}$ Functions $(X, T)$. Then $v+u \in \mathrm{C}_{0}$ Functions $(X, T)$. The theorem is a consequence of (5) and (54).

(57) Let us consider a non empty topological space $X$, a normed linear topological space $T$, a real number $a$, and an element $u$ of RealVectSpace((the carrier of $X), T)$. Suppose $u \in \mathrm{C}_{0}$ Functions $(X, T)$. Then $a \cdot u \in \mathrm{C}_{0} \operatorname{Functions}(X$, $T)$. The theorem is a consequence of (5) and (55).

(58) Let us consider a non empty topological space $X$, and a normed linear topological space $T$. Then $\mathrm{C}_{0}$ Functions $(X, T)$ is linearly closed.

Let $X$ be a non empty topological space and $T$ be a normed linear topological space. Let us note that $\mathrm{C}_{0}$ Functions $(X, T)$ is non empty and linearly closed.

The functor $\mathrm{RV}_{\mathrm{SP}} \mathrm{C}_{0}$ Functions $(X, T)$ yielding a real linear space is defined by the term

(Def. 11) $\left\langle\mathrm{C}_{0}\right.$ Functions $(X, T)$, Zero $\left(\mathrm{C}_{0}\right.$ Functions $(X, T)$, RealVectSpace $(($ the carrier of $X), T)), \operatorname{Add}\left(\mathrm{C}_{0}\right.$ Functions $(X, T)$, RealVectSpace $(($ the carrier of $\left.X), T)\right)$, $\operatorname{Mult}\left(\mathrm{C}_{0}\right.$ Functions $(X, T)$, RealVectSpace $(($ the carrier of $\left.\left.X), T)\right)\right\rangle$.

Now we state the propositions:

(59) Let us consider a non empty topological space $X$, and a normed linear topological space $T$. Then $\operatorname{RV}_{\mathrm{SP}} \mathrm{C}_{0} \operatorname{Functions}(X, T)$ is a subspace of RealVectSpace((the carrier of $X), T)$.

(60) Let us consider a non empty topological space $X$, a normed linear topological space $T$, and a set $x$. Suppose $x \in \mathrm{C}_{0}$ Functions $(X, T)$. Then $x \in \operatorname{BdFuncs}(($ the carrier of $X), T)$.

Proof: Consider $f$ being a function from the carrier of $X$ into the carrier of $T$ such that $f=x$ and $f$ is continuous and there exists a non empty subset $Y$ of $X$ such that $Y$ is compact and support $f \subseteq Y$. Consider $Y$ being a non empty subset of $X$ such that $Y$ is compact and support $f \subseteq Y$. Consider $K$ being a real number such that $0 \leqslant K$ and for every point $x$ of $X$ such that $x \in Y$ holds $\|f(x)\| \leqslant K$. For every element $x$ of $X$, $\|f(x)\| \leqslant K$.

Let $X$ be a non empty topological space and $T$ be a normed linear topological space. The functor $\operatorname{NormC}_{0} \operatorname{Functions}(X, T)$ yielding a function from $\mathrm{C}_{0}$ Functions $(X, T)$ into $\mathbb{R}$ is defined by the term

(Def. 12) BdFuncsNorm( (the carrier of $X), T)\left\lceil\mathrm{C}_{0}\right.$ Functions $(X, T)$.

The functor $\operatorname{NormSp}_{\mathrm{C}_{0}}$ Functions $(X, T)$ yielding a normed structure is defined by the term

(Def. 13) $\left\langle\mathrm{C}_{0}\right.$ Functions $(X, T)$, Zero $\left(\mathrm{C}_{0} \operatorname{Functions}(X, T)\right.$, RealVectSpace $(($ the carrier of $X), T)), \operatorname{Add}\left(\mathrm{C}_{0}\right.$ Functions $(X, T)$, RealVectSpace $(($ the carrier of $X), T)$ ), $\operatorname{Mult}\left(\mathrm{C}_{0}\right.$ Functions $(X, T)$, RealVectSpace $(($ the carrier of $\left.X), T)\right)$, 
$\left.\operatorname{Norm} \mathrm{C}_{0} \operatorname{Functions}(X, T)\right\rangle$.

Let us note that $\operatorname{NormSp}_{\mathrm{C}_{0}} \operatorname{Functions}(X, T)$ is strict and non empty.

Now we state the proposition:

(61) Let us consider a non empty topological space $X$, a normed linear topological space $T$, and a set $x$. Suppose $x \in \mathrm{C}_{0}$ Functions $(X, T)$. Then $x \in$ the continuous functions of $X$ and $T$.

Let us consider a non empty topological space $X$ and a normed linear topological space $T$. Now we state the propositions:

(62) $0_{\mathrm{RV}_{\mathrm{SP}} \mathrm{C}_{0} \operatorname{Functions}(X, T)}=X \longmapsto 0_{T}$.

(63) $0_{\mathrm{NormSp}_{\mathrm{C}_{0}}}$ Functions $(X, T)=X \longmapsto 0_{T}$. The theorem is a consequence of $(62)$.

(64) Let us consider a non empty topological space $X$, a normed linear topological space $T$, points $x_{1}, x_{2}$ of $\operatorname{NormSp}_{\mathrm{C}_{0}} \operatorname{Functions}(X, T)$, and points $y_{1}, y_{2}$ of the real normed space of bounded functions from the carrier of $X$ into $T$. If $x_{1}=y_{1}$ and $x_{2}=y_{2}$, then $x_{1}+x_{2}=y_{1}+y_{2}$.

(65) Let us consider a non empty topological space $X$, a normed linear topological space $T$, a real number $a$, a point $x$ of $\operatorname{NormSp}_{\mathrm{C}_{0}} \operatorname{Functions}(X, T)$, and a point $y$ of the real normed space of bounded functions from the carrier of $X$ into $T$. If $x=y$, then $a \cdot x=a \cdot y$.

(66) Let us consider a real number $a$, a non empty topological space $X$, a normed linear topological space $T$, and points $F, G$ of $\operatorname{NormSp}_{\mathrm{C}_{0}} \operatorname{Functions}(X$, $T)$. Then

(i) $\|F\|=0$ iff $F=0_{\operatorname{NormSp}_{\mathrm{C}_{0}}} \operatorname{Functions}(X, T)$, and

(ii) $\|a \cdot F\|=|a| \cdot\|F\|$, and

(iii) $\|F+G\| \leqslant\|F\|+\|G\|$.

ProOF: $\|F\|=0$ iff $F=0_{\operatorname{NormSp}_{\mathrm{C}_{0}} \operatorname{Functions}(X, T)} \cdot\|a \cdot F\|=|a| \cdot\|F\|$. $\|F+G\| \leqslant\|F\|+\|G\|$ by (60), (64) [6, (21)].

(67) Let us consider a non empty topological space $X$, and a normed linear topological space $T$. Then $\operatorname{NormSp}_{\mathrm{C}_{0}} \operatorname{Functions}(X, T)$ is real normed spacelike.

Let $X$ be a non empty topological space and $T$ be a normed linear topological space. Let us note that $\operatorname{NormSp}_{\mathrm{C}_{0}} \operatorname{Functions}(X, T)$ is reflexive, discernible, real normed space-like, vector distributive.

And let us observe that $\operatorname{NormSp}_{\mathrm{C}_{0}} \operatorname{Functions}(X, T)$ is scalar distributive, scalar associative, scalar unital, Abelian, add-associative, right zeroed, and right complementable.

Now we state the proposition: 
(68) Let us consider a non empty topological space $X$, and a normed linear topological space $T$. Then $\operatorname{NormSp}_{\mathrm{C}_{0}} \operatorname{Functions}(X, T)$ is a real normed space.

\section{REFERENCES}

[1] Grzegorz Bancerek, Czesław Byliński, Adam Grabowski, Artur Korniłowicz, Roman Matuszewski, Adam Naumowicz, Karol Pąk, and Josef Urban. Mizar: State-of-the-art and beyond In Manfred Kerber, Jacques Carette, Cezary Kaliszyk, Florian Rabe, and Volker Sorge, editors, Intelligent Computer Mathematics, volume 9150 of Lecture Notes in Computer Science, pages 261-279. Springer International Publishing, 2015. ISBN 978-3319-20614-1. doi 10.1007/978-3-319-20615-8_17.

[2] Grzegorz Bancerek, Czesław Byliński, Adam Grabowski, Artur Korniłowicz, Roman Matuszewski, Adam Naumowicz, and Karol Pak. The role of the Mizar Mathematical Library for interactive proof development in Mizar. Journal of Automated Reasoning, 61(1):9-32, 2018. do1 $10.1007 /$ s10817-017-9440-6

[3] Bruce K. Driver. Analysis Tools with Applications. Springer, Berlin, 2003.

[4] Takaya Nishiyama, Keiji Ohkubo, and Yasunari Shidama. The continuous functions on normed linear spaces Formalized Mathematics, 12(3):269-275, 2004.

[5] Laurent Schwartz. Théorie des ensembles et topologie, tome 1. Analyse. Hermann, 1997.

[6] Yasumasa Suzuki. Banach space of bounded real sequences Formalized Mathematics, 12 (2):77-83, 2004.

[7] Hiroshi Yamazaki. Functional sequence in norm space. Formalized Mathematics, 28(4): 263-268, 2020. doi $10.2478 /$ forma-2020-0023 